\title{
Supercontinuum Generation with GHz Repetition Rate Femtosecond-Pulse Fiber-Amplified VECSELs
}

\author{
C. Robin Head ${ }^{1, *}$, Ho-Yin Chan ${ }^{2, *}$, James S. Feehan ${ }^{2}$, David P. Shepherd ${ }^{2}$, Shaif-ul Alam ${ }^{2}$, Anne C. Tropper ${ }^{1}$, \\ Jonathan H. V. Price ${ }^{2}$ and Keith G. Wilcox ${ }^{1}$
}

\begin{abstract}
We report supercontinuum generation using a mode-locked VECSEL emitting 400-fs pulses at a 3-GHz repetition rate, amplified with a cascaded ytterbium-doped fiber amplifier system up to $40 \mathrm{~W}$ of average power. The pulses were then recompressed to their original duration via a high throughput transmission grating compressor, and used to generate supercontinuum in two samples of photonic crystal fiber (PCF); an all-normal dispersion PCF, and a PCF with a zero dispersion wavelength of $1040 \mathrm{~nm}$, creating $20 \mathrm{~dB}$ spectral bandwidths of $200 \mathrm{~nm}$ and $280 \mathrm{~nm}$ respectively.
\end{abstract}

Index Terms-Fiber amplifiers, vertical-external-cavity surface-emitting laser (VECSEL), supercontinuum generation, GHz repetition rate

\section{INTRODUCTION}

$\mathbf{T}$ HE generation of supercontinuum in photonic crystal fiber $(\mathrm{PCF})$ has found applications in a wide range of research areas such as telecommunications, metrology and spectroscopy. Typical laser sources used to generate supercontinuum are pulsed solid-state or fiber lasers that operate at repetition rates up to approximately $100 \mathrm{MHz}$. While these low-repetition-rate sources easily produce spectrally broad supercontinuum due to their high peak power, higher repetition rates are desirable for frequency combs, as the increased mode spacing leads to more energy per frequency mode, and it is possible to isolate individual modes using a simple diffraction grating [1], [2]. The supercontinuum generated for frequency combs should, however, be coherent, which typically requires sub-100 fs pulse durations when using PCF pumped close to its zero dispersion wavelength (ZDW) [3]. This limit can be stretched as Stumpf et al. have demonstrated with 170fs pulses at a wavelength of 1.5 micron using state-of-theart dispersion-flattened, polarization-maintaining, highly nonlinear fiber [4]. Alternatively supercontinuum with a high degree of coherence can be generated using all-normal dispersion PCF with pulse durations of several hundred femtoseconds [5], [6]. Mode-locked (ML) vertical-external-cavity surface-emitting semiconductor lasers (VECSELs) typically work at $\mathrm{GHz}$ repetition rates [7], emit near-transform-limited pulses [8] with pulse durations down to $60 \mathrm{fs}$ [9], and have

*robin.head@soton.ac.uk, hyc1g11@ orc.soton.ac.uk

[1] School of Physics and Astronomy, University of Southampton, Southampton SO17 1BJ, UK

[2] Optoelectronics Research Centre, University of Southampton, Southampton SO17 1BJ, UK

Manuscript received October 12, 2012; revised XXXX XX, 2012. Copyright (c) 2012 IEEE. Personal use of this material is permitted. However, permission to use this material for any other purposes must be obtained from the IEEE by sending a request to pubspermissions@iee.org. reached average powers up to $5.1 \mathrm{~W}$ with sub-picosecond pulses [10]. Furthermore the mode-locking dynamics allows continuous repetition rate tuning over a range of several $\mathrm{GHz}$ by changing the length of the external cavity [11], [12]. Thus VECSELs are potentially flexible sources for coherent $\mathrm{GHz}$ supercontinuum generation. However the direct generation of both sub-100 fs pulses and high average output power in VECSELs has not been achieved yet. Sub-picosecond pulsed fiber amplifier systems have reached output powers of $830 \mathrm{~W}$ at a $78 \mathrm{MHz}$ repetition rate with a $640 \mathrm{fs}$ pulse duration using a chirped pulse amplification architecture [13]. More recently a fiber master oscillator power amplifier (MOPA) system has achieved $110 \mathrm{~W}$ at a $1.3 \mathrm{GHz}$ repetition rate with a 890 fs pulse duration requiring only modest pulse stretching [14]. As the repetition rate increases, the pulse energy decreases and the linear amplification regime can be used to correspondingly higher average powers before pulse stretching becomes neccessary. Fiber MOPA systems provide a complementary technology to ML-VECSELs to enable high power $\mathrm{GHz}$ amplification and supercontinuum generation. In previous work Dupriez et al. reported a femtosecond seeded MOPA system where average powers of $53 \mathrm{~W}$ were reached [15]. However only a few hundred milliwatts of the output power could be used, to demonstrate that it was possible to cleanly compress the pulses, due to the power handling of the aluminum reflection grating compressor.

Recently Chamorovskiy et al. have generated a supercontinuum with a VECSEL MOPA system where a $500 \mathrm{~m}$ long $\mathrm{GeO}_{2}$-codoped silica fiber, driven by a $1.57 \mu \mathrm{m}$ VECSEL MOPA producing 15.5 ps pulses, was used. [16].

Here we report a VECSEL MOPA system producing $40 \mathrm{~W}$ of average power. A $70 \%$ throughput grating compressor was used giving $400 \mathrm{fs}$ pulses. These pulses are then used to generate supercontinuum in two samples of PCF; an all-normal dispersion PCF, and a PCF with a zero dispersion wavelength of $1040 \mathrm{~nm}$.

\section{EXPERIMENTAL SET-UP}

The amplified pulse source, shown in Figure 1, consists of distinct sections; The VECSEL, the cascaded fiber amplifier system and the compressor. The compressed output was then launched into the PCF to generate supercontinuum. We describe the VECSEL in section II-A followed by the fiber amplifier and pulse compression system in section II-B. 


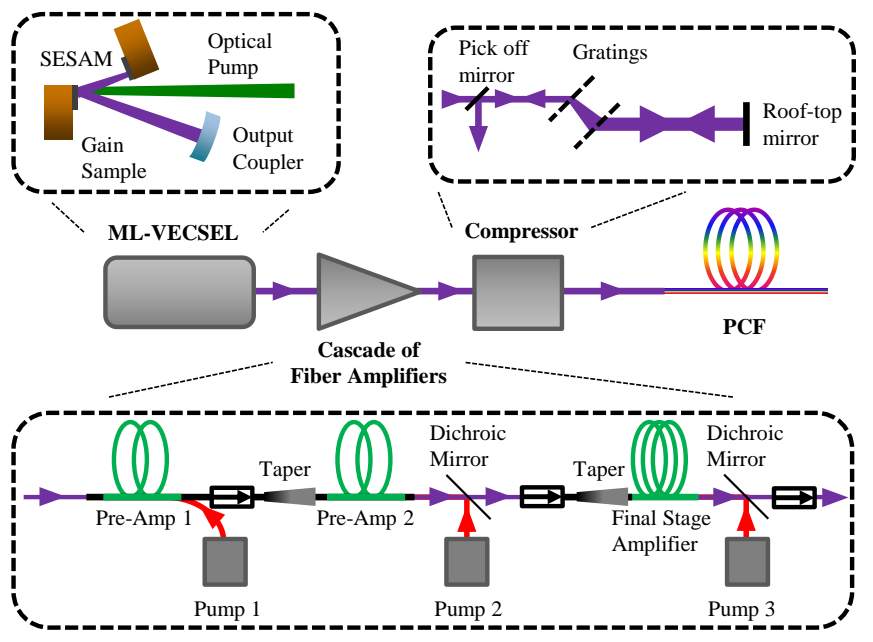

Fig. 1. Schematic of the experimental set-up. The VECSEL was used as a seed laser for a three-stage ytterbium-doped fiber amplifier system. The amplified pulses were recompressed with a high throughput grating compressor and free space launched into the PCF.

\section{A. VECSEL}

The ML-VECSEL in this experiment consists of the gain structure, a Semiconductor Saturable Absorber Mirror (SESAM) and a spherical output coupler. The gain structure consists of six strain-balanced InGaAs quantum wells designed for $1025 \mathrm{~nm}$, grown on top of a 27.5 pair AlAs/GaAs distributed Bragg reflector (DBR). The SESAM is used to mode-lock the laser and has a single InGaAs quantum well designed for $1025 \mathrm{~nm}$, on top of a DBR designed at $1040 \mathrm{~nm}$. The design and optical properties of the gain structure and SESAM have been described in [7]. The VECSEL is optically pumped using an $830 \mathrm{~nm}$ fiber coupled diode laser, which is focused to a $60 \mu \mathrm{m}$ radius spot on the gain structure. A V-shaped cavity is formed by a $0.3 \%$ output coupler with a radius of curvature of $50 \mathrm{~mm}$, the gain structure which acts as a plane folding mirror, and the SESAM as the cavity end mirror. The cavity is designed to give a fundamental laser mode with a $60 \mu \mathrm{m}$ radius waist on the gain and a $20 \mu \mathrm{m}$ radius waist on the SESAM, ensuring that the SESAM is saturated more strongly than the gain. The gain structure is mounted on a water-cooled temperature-controlled copper block. Neither the gain nor the SESAM structure has been processed, limiting the maximum average output power [17]. All components are directly mounted on a single Invar plate to reduce misalignment due to vibrations or thermal drift and thus improve long-term mode-locked stability.

\section{B. Amplifier System}

The MOPA system used in this experiment is similar to the set-up used by Chen et al. [18], [19] but with a VECSEL as master oscillator. The system is based on polarizationmaintaining large mode area (LMA) fiber technology, ensuring a robust and stable amplifier system. The first and second pre-amplifiers are ytterbium-doped fibers with core diameters of $5 \mu \mathrm{m}$ and $25 \mu \mathrm{m}$ and lengths of $2 \mathrm{~m}$ and $2.7 \mathrm{~m}$ respectively. Both pre-amplification stages are reverse pumped using
$975 \mathrm{~nm}$ fiberized diodes. The small core fiber in the first amplifier was chosen in order to maintain a good signal-tonoise ratio and to provide high gain with the low-power wavelength division multiplexed coupled pump-diode. The second preamplifier had a larger core to reduce nonlinear effects and had sufficient output power to saturate the final amplifier. We used fiber lengths long enough to efficiently absorb the pump power but short enough to avoid gain narrowing [20]. Following each pre-amplification stage the signal is passed through an isolator. After pre-amplification the signal is freespace-coupled into the final-stage amplifier. The final-stage amplifier fiber, similar to that used in the second pre-amplifier, is a $3 \mathrm{~m}$ length of double-clad LMA ytterbium-doped fiber, which is reverse pumped with a high power fiberized diode laser source (Laserline LDM 200-200) at $976 \mathrm{~nm}$. The pump launch efficiency for the final two amplifiers was estimated to be $\sim 70 \%$. Single mode operation $\left(\mathrm{M}^{2}<1.1\right)$ was achieved from these few-mode LMA fibers by pre-tapering the input to act as a mode filter as described by Chen et al. [18]. Following the final stage amplifier, the amplified signal passes through an isolator and a high throughput compressor (Ibsen FSTG-PCG-1250-1064 gratings) with a grating separation of approximately $2 \mathrm{~cm}$. The amplified and compressed VECSEL signal is directly free-space coupled into PCF. To improve thermal handling, and thus allow for a higher average power to be launched, the end-caps of the PCF are collapsed and polished. The two different PCFs used in this experiment are a $10 \mathrm{~m}$ long all-normal dispersion PCF (NL-1050-NEG-1) with a dispersion minimum at $1050 \mathrm{~nm}$; and a $1 \mathrm{~m}$ long PCF (SC5.0-1040) with a ZDW of $1040 \mathrm{~nm}$, closely matched to the wavelength of amplified signal.

\section{EXPERIMENTAL RESULTS}

\section{A. Amplifier System}

The target wavelength for the VECSEL seed is $1040 \mathrm{~nm}$ to achieve optimal amplification in the ytterbium fiber amplifier system. For this particular combination of gain and SESAM wafers, a heat sink temperature of $15^{\circ} \mathrm{C}$ and pump power of $1280 \mathrm{~mW}$ produced a laser spectrum centered at $1040 \mathrm{~nm}$ with a Full Width Half Maximum (FWHM) of $2.85 \mathrm{~nm}$, corresponding to a train of near transform-limited 400 fs pulses at a repetition rate of $3 \mathrm{GHz}$ and an average output power of $20 \mathrm{~mW}$. Figure 2 shows the optical spectrum and intensity autocorrelation of the VECSEL output.

The average power of the signal after the first two preamplification stages was $1.5 \mathrm{~W}$ with pulse durations of $1.7 \mathrm{ps}$. Pump powers of $210 \mathrm{~mW}$ and $5.4 \mathrm{~W}$ were used for the first and second stages respectively. The pulse duration after the final stage power amplifier is 3 ps at an average power of up to $40 \mathrm{~W}$, limited by pump absorption. The pulse elongation was predominantly due to the fiber dispersion in the amplifier system. These average powers were still in the linear amplification regime and so no significant spectral broadening could be observed and no complex phase structure was added by the amplifier chain. Thus it was possible to recompress the pulses to their original duration of $400 \mathrm{fs}$, as shown in Figure 3. Scaling the average power to higher powers would enable 


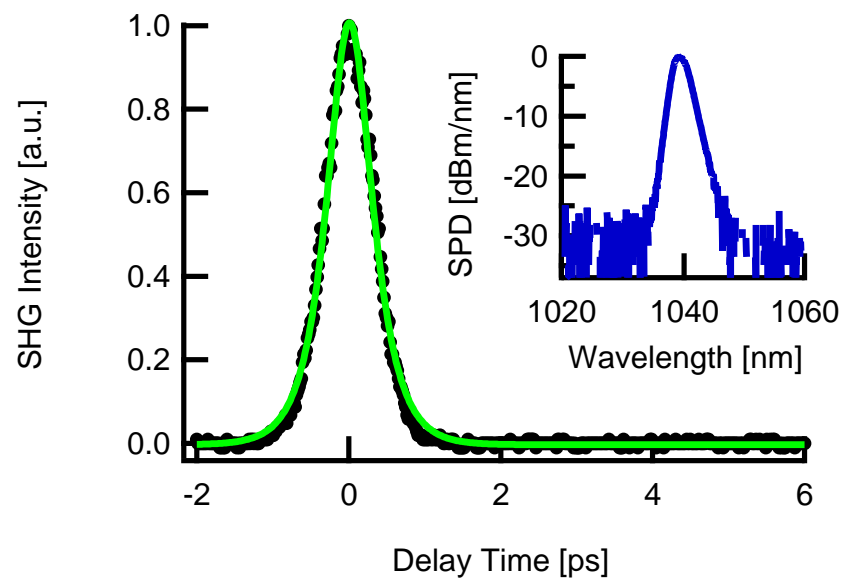

Fig. 2. Second harmonic generation (SHG) intensity autocorrelation of the pulse (black) with a sech ${ }^{2}$ fit (green). The inset shows the normalized spectral power density (SPD) of the spectrum of the ML-VECSEL. The pulse duration of the VECSEL is $400 \mathrm{fs}$ and the center wavelength of the VECSEL is at $1040 \mathrm{~nm}$ with a full width at half maximum of $2.85 \mathrm{~nm}$.

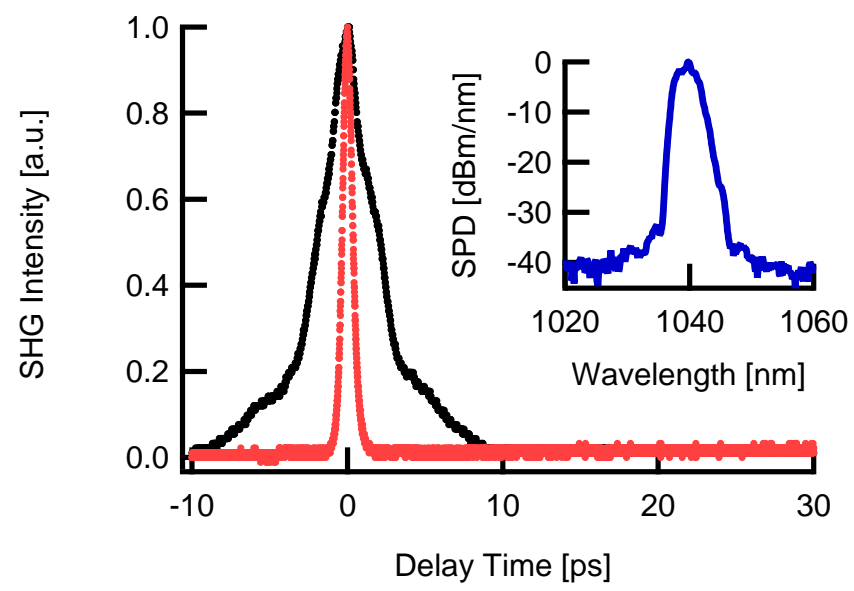

Fig. 3. Autocorrelations of the uncompressed (black) and compressed (red) pulse. The inset shows the spectrum after the final stage amplifier. The uncompressed pulse duration is $\sim 3$ ps: the recompressed pulses have a $400 \mathrm{fs}$ duration.

parabolic pulse amplification and allow for pulse compression to sub-original durations.

\section{B. Supercontinuum Generation}

The recompressed 400 fs pulses are launched into the two PCFs in turn, achieving in each case a transmission efficiency of $\sim 50 \%$. The maximum average powers measured after the PCFs are $2.5 \mathrm{~W}$ and $3.9 \mathrm{~W}$ for the PCF pumped closely to its ZDW and all-normal dispersion PCF respectively, limited by thermal management of the passively cooled launch into the PCF. Figure 4 shows the spectra of the supercontinuum generated with the VECSEL MOPA system. The $20 \mathrm{~dB}$ bandwidth of the supercontinuum created with the all-normal dispersion PCF is $200 \mathrm{~nm}$. We expect this supercontinuum to have a high degree of coherence [5], [6]. The pulse predominantly acquires a linear chirp and it should be compressible with appropriate dispersion compensation. A

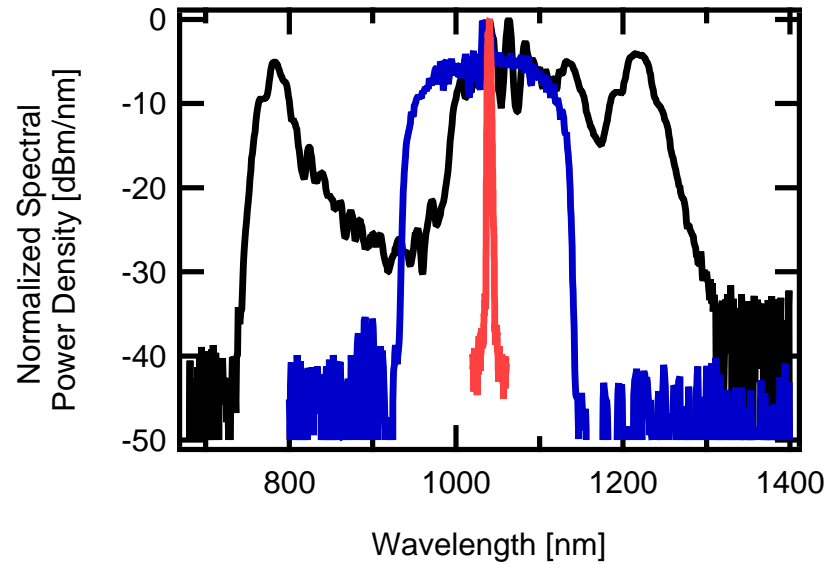

Fig. 4. Measured input spectrum (red) and supercontinuum spectra produced with the all-normal dispersion PCF (blue) and the PCF with a ZDW of $1040 \mathrm{~nm}$ (black). The $20 \mathrm{~dB}$ bandwidth of the all-normal dispersion PCF supercontinuum is $200 \mathrm{~nm}$ and $280 \mathrm{~nm}$ for the PCF pumped closely to its ZDW.

$280 \mathrm{~nm}$ 20-dB-bandwidth supercontinuum is measured for the PCF with a ZDW of $1040 \mathrm{~nm}$. The supercontinuum had spectral components between $750 \mathrm{~nm}$ and $1300 \mathrm{~nm}$. The dip at about $900 \mathrm{~nm}$ is typical for PCFs with a ZDW and is due to the relation between the pump wavelength and the dispersive properties of the PCF, however for a detailed explanation a numerical model would be necessary [3]. In this case the supercontinuum is thought to be temporally incoherent as the pulse duration launched into the PCF is significantly above $100 \mathrm{fs}$ [3].

\section{CONCLUSION}

We have demonstrated that the combination of femtosecond ML-VECSELs and fiber amplifiers can be used to generate supercontinuum at multi-GHz repetition rates at average powers of several Watts in a robust and practical system. Supercontinuum with a $20 \mathrm{~dB}$ bandwidth width of $280 \mathrm{~nm}$ and an average output power of $2.5 \mathrm{~W}$ was achieved with a PCF pumped close to its ZDW. For supercontinuum generated with all-normal dispersion PCF a $20 \mathrm{~dB}$ spectral bandwidth of $200 \mathrm{~nm}$ was achieved with an average output power of $3.9 \mathrm{~W}$. The VECSEL-MOPA produced up to $40 \mathrm{~W}$ output power at a $3 \mathrm{GHz}$ repetition rate. The pulses were re-compressed to 400 fs durations using a high throughput transmission grating compressor. High power supercontinuum generation has been reported by Chen et al. [19] and we aim to use techniques developed in that work along with PCF with optimized dispersion characteristics to generate higher average power broader bandwidth supercontinuum in the future. A VECSEL MOPA system employing a shorter seed pulse and optimized amplifier design should enable parabolic regime amplification to produce shorter pulses which could be used to produce octave-spanning coherent supercontinuum.

\section{ACKNOWLEDGMENT}

C. Robin Head and Ho-Yin Chan have contributed equally to this work. We thank R. Malik for advice and in- 
put. This work was undertaken with funding from EPSRC (EP/G059268/1 and EP/I02798X/1). J. H.V. Price was supported by a RAEng/EPSRC Fellowship and K. G. Wilcox holds an EPSRC Early Career Fellowship.

\section{REFERENCES}

[1] T. M. Fortier, A. Bartels, and S. A. Diddams, "Octave-spanning Ti:sapphire laser with a repetition rate $>1 \mathrm{GHz}$ for optical frequency measurements and comparisons," Optics Letters, vol. 31, no. 7, pp. 1011-1013, 2006.

[2] A. Bartels, D. Heinecke, and S. A. Diddams, "10-GHz Self-Referenced Optical Frequency Comb,” Science, vol. 326, p. 681, 2009.

[3] J. M. Dudley, G. Genty, and S. Coen, "Supercontinuum generation in photonic crystal fiber," Reviews of Modern Physics, vol. 78, no. 4, pp. 1135-1184, 2006.

[4] M. C. Stumpf, S. Pekarek, A. E. H. Oehler, T. Südmeyer, J. M. Dudley, and U. Keller, "Self-referenceable frequency comb from a 170-fs, 1.5m solid-state laser oscillator," Applied Physics B, vol. 99, pp. 401-408, 2010.

[5] A. M. Heidt, "Pulse preserving flat-top supercontinuum generation in allnormal dispersion photonic crystal fibers," J. Opt. Soc. Am B, vol. 27, no. 3 , pp. 550-559, 2010.

[6] K. K. Chow, Y. Takushima, C. Lin, C. Shu, and A. Bjarklev, "Flat supercontinuum generation based on normal dispersion nonlinear photonic crystal fibre," Electronics Letters, vol. 42, pp. 989-990, 2006.

[7] A. C. Tropper, A. H. Quarterman, and K. G. Wilcox, "Ultrafast VerticalExternal-Cavity Surface-Emitting Semiconductor Lasers," Advances in Semiconductor Lasers, vol. 86, pp. 269-300, 2012.

[8] K. G. Wilcox, Z. Mihoubi, G. J. Daniell, S. Elsemere, A. Quarterman, I. Farrer, D. A. Ritchie, and A. Tropper, "Ultrafast optical Stark modelocked semiconductor laser," Optics Letters, vol. 33, no. 23, pp. 27972799, 2008.

[9] A. H. Quarterman, K. G. Wilcox, V. Apostolopoulos, Z. Mihoubi, S. P. Elsemere, I. Farrer, D. A. Ritchie, and A. Tropper, "A passively mode-locked external-cavity semiconductor laser emitting 60-fs pulses," Nature Photonics, vol. 3, pp. 729-731, 2009.

[10] M. Scheller, T.-L. Wang, B. Kunert, W. Stolz, S. W. Koch, and J. V. Moloney, "Passively modelocked VECSEL emitting $682 \mathrm{fs}$ pulses with $5.1 \mathrm{~W}$ of average output power," Electronics Letters, vol. 48, pp. 588 $589,2012$.

[11] K. G. Wilcox, A. H. Quarterman, H. E. Beere, D. A. Ritchie, and A. C. Tropper, "Variable repetition frequency femtosecond-pulse surface emitting semiconductor laser," Applied Physics Letters, vol. 99, p. 131107, 2011.

[12] O. D. Sieber, V. J. Wittwer, M. Mangold, M. Hoffmann, T. Golling, M Sdmeyer, and U. Keller, "Femtosecond VECSEL with tunable multigigahertz repetition rate," Optics Express, vol. 19, no. 23, pp. 23538 $23543,2011$.

[13] T. Eidam, S. Hanf, E. Seise, T. V. Andersen, T. Gabler, C. Wirth, T. Schreiber, J. Limpert, and A. Tünnermann, "Femtosecond fiber CPA system emitting $830 \mathrm{~W}$ average output power," Optics Letters, vol. 35, no. 2, pp. 94-96, 2010.

[14] Z. Zhao, B. M. Dunham, I. Bazarov, and F. W. Wise, "Generation of $110 \mathrm{~W}$ infrared and $65 \mathrm{~W}$ green power from a 1.3-GHz sub-picosecond fiber amplifier,' Optics Express, vol. 20, no. 5, pp. 4850-4855, 2012.

[15] P. Dupriez, C. Finot, A. Malinowski, J. K. Sahu, J. Nilsson, D. J. Richardson, K. G. Wilcox, H. D. Foreman, and A. C. Tropper, "Highpower, high repetition rate picosecond and femtosecond sources based on Yb-doped fiber amplification of VECSELs," Optics Express, vol. 14, no. 21, pp. 9611-9616, 2006.

[16] A. Chamorovskiy, J. Kerttula, J. Rautiainen, and O. G. Okhotnikov, "Supercontinuum generation with amplified $1.57 \mu \mathrm{m}$ picosecond semiconductor disk laser," Electronics Letters, vol. 48, no. 16, 2012.

[17] S. Calvez, J. E. Hastie, A. J. Kemp, N. Laurand, and M. D. Dawson, Thermal management, structure design and integration considerations for vecsels, in Semiconductor Disk Lasers: Physics and Technology, O. G. Okhotnikov, Ed. Weinheim, Germany: Wiley-VCH Verlag GmbH \& Co. KGaA, 2010.

[18] K. K. Chen, J. H. V. Price, S. Alam, J. R. Hayes, D. Lin, A. Malinowski, and D. J. Richardson, "Polarisation maintaining 100W Yb-fiber MOPA producing $\mu \mathrm{J}$ pulses tunable in duration from 1 to 21 ps," Optics Express, vol. 18 , no. 14 , pp. 14385-14394, 2010.
[19] K. K. Chen, J. H. V. Price, S. Alam, J. R. Hayes, D. Lin, A. Malinowski, C. Codemard, D. Ghosh, M. Pal, S. K. Bhadra, and D. J. Richardson, "Picosecond fiber MOPA pumped supercontinuum source with 39 W output power," Optics Express, vol. 18, no. 6, pp. 5426-5432, 2010.

[20] F. He, J. H. V. Price, K. T. Vu, A. Malinowski, J. K. Sahu, and D. J. Richardson, "Optimisation of cascaded Yb fiber amplifier chains using numerical-modelling," Optics Express, vol. 14, pp. 12 846-12 858, 2006. 